\title{
Pain Research
}

National Cancer Institute

\section{Source}

National Cancer Institute. Pain Research. NCI Thesaurus. Code C16141.

Research as to the cause of pain and the treatment thereof 